\title{
Effects of Chain Structure and Network Constitution on Segmental Orientation in Deformed Amorphous Networks
}

\author{
Burak Erman* and Ivet Bahar
}

School of Engineering, Bogazici University, Bebek 80815 Istanbul, Turkey. Received July 8, 1987; Revised Manuscript Received August 20, 1987

\begin{abstract}
Segmental orientation in deformed networks is analyzed in terms of configurational properties of constituent chains and of the network structure. Configurational evaluation by using the rotational isomeric state approximation with the polyethylene chain as an example shows that orientation depends strongly on (i) the direction of the investigated label relative to a chain bond, (ii) chain length, (iii) label location along the chain, (iv) configurational perturbations due to presence of a label along the chain, and (v) polydispersity. A molecular model of the network is required for the analysis of the effect of network constitution on segmental orientation. The constrained junction theory of rubber elasticity is employed to estimate the contributions to segmental orientation from the network structure.
\end{abstract}

\section{Introduction}

The directions of chain segments in an isotropic network at equilibrium are randomly distributed. When the network is macroscopically distorted, the orientations of segments become anisotropic. The state of anisotropy under strain may accurately be characterized by spectroscopic techniques with which the orientations of segments, or of vectors affixed to specific segments, can directly be measured. Infrared dichroism, ${ }^{2} \mathrm{H}$ NMR spectroscopy, fluorescence polarization, and wide-angle $\mathrm{X}$-ray scattering are four of the techniques that conveniently describe segmental orientation in deformed networks. Critical interpretation of experimental data requires, however, a molecular model of the network relating macroscopic strain to network topology and to configurational features of individual chains. The simplest and the most commonly employed model is for a network with affinely deforming freely jointed chains. The expression for the orientation function, $S$, for such a network in simple tension is

$$
S=(1 / 5 N)\left(\lambda^{2}-\lambda^{-1}\right)
$$

where $N$ is the number of bonds in the network chain and $\lambda$ is the extension ratio. The orientation function is defined by the ensemble-averaged second Legendre polynomial,

$$
S=\left(3\left\langle\cos ^{2} \theta\right\rangle-1\right) / 2
$$

where $\left\langle\cos ^{2} \theta\right\rangle$ is the mean-squared cosine of the angle between a bond and the direction of stretch. For a real chain $N$ represents the number of Kuhn segments. Although eq 1 reflects the basic features of segmental orientation in a network, it is insufficient in describing the behavior of real networks. The precision obtained in measurements of orientation by recent spectroscopic techniques calls for a quantitatively more accurate expression for segmental orientation. In this respect, the shortcomings of eq 1 are severalfold. First, the front factor suffers from an arbitrariness in the choice of the number of equivalent bonds in a Kuhn segment of a freely jointed chain. Second, this expression describes the orientation of vectors that are along the bonds. However, experiments most often measure the orientations of vectors that are fixed at a specific angle to the bond vector. Configurational properties of the constituent chains are required for the calculation of the orientation of different vectors affixed to a segment. Third, the assumption of affine chain deformation is too approximate for describing the behavior of real networks.

The specific aim of the present study is to show possible improvement of eq 1 for a more realistic and quantitative description of segmental orientation in polymeric media. According to the discussion in the preceding paragraph, improvements may be made independently for the configurational factor, $1 / 5 N$, and the strain function, $\lambda^{2}-1 / \lambda$. In a recent study, the latter was formulated according to the constrained junction theory of rubber elasticity. ${ }^{1}$ Predictions based on this formulation were shown ${ }^{2}$ to be in qualitative agreement with orientation measurements of polyisoprene networks in simple tension. In the present study more emphasis is placed on the evaluation of the configurational factor for a real chain. In the first section below, the theory that leads to improvements in the configurational factor of eq 1 is reviewed. This theory $y^{3,4}$ is based on the configurational calculations of the orientations of a specific vector affixed to one (or more) locations along a chain whose two ends are fixed in space. The property calculated in this manner results from intramolecular effects only. In order to eliminate contributions from intermolecular correlations, a phantom network is adopted. A phantom network is, by definition, topologically equivalent to a real network in which all intermolecular correlations are eliminated. ${ }^{5}$ The rotational isomeric state formalism is adopted for calculating the configurational factor for segmental orientation. Detailed calculations are performed for the polymethylene chain. This system is chosen for the simplicity of the molecular structure. However, the generality of the method described allows for calculations for any other polymeric system. In the second section, contributions from network topology and intermolecular correlations to segmental orientation are considered. In the final section, the predictions of the theory are discussed in relation to experimental work in this field.

\section{Evaluation of the Configurational Factor}

A chain of a network is depicted in Figure 1a by the curve $O A$. $O X Y Z$ represents a laboratory fixed coordinate system. The ends $O$ and $A$ of the chain are assumed to be cross-link points to which other chains of the network are attached. $\mathbf{r}$ is the end-to-end vector of the chain at a given instant. $\mathbf{m}_{i}$ is a unit vector affixed to the chain at bond $i$. It represents the "label" observed by the various experimental techniques. Depending on the type of experiment, $\mathbf{m}_{i}$ may be a single vector at a specified location or it may be repeated at several locations along the chain. Similarly, each or only a few of the chains may contain such labels. The calculation of the average orientation of vectors in a network proceeds in two steps. ${ }^{5}$ First, the two ends of the chain of interest are kept fixed at a separation $\mathbf{r}$ and the average orientation of the vector $\mathbf{m}_{i}$ with respect to the laboratory fixed $X$-axis, for example, is determined. In the second step, the value obtained for the fixed $\mathbf{r}$ is averaged over the ensemble of chains in the deformed 


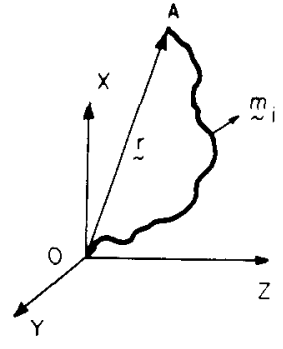

(a) (b)
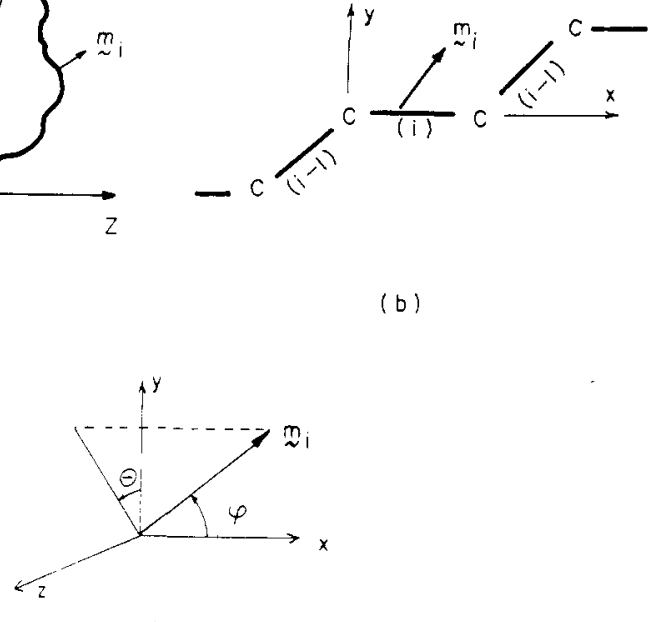

(c)

Figure 1. (a) Chain of the network with end-to-end vector $\mathbf{r}$ and a vector $\mathbf{m}_{i}$ affixed to location $i . O X Y Z$ is the laboratory fixed coordinate system. (b) A short sequence of $\mathrm{C}-\mathrm{C}$ bonds. Letters in parentheses indicate bond number. $\mathbf{m}_{i}$ is rigidly affixed to bond $i$. $x y z$ is the molecule fixed coordinate system at bond $i$. The $y$-axis is in the plane of bonds $i-1$ and $i$. The $z$-axis (not shown) completes a right-handed system. (c) Polar, $\phi$, and azimuthal, $\theta$, angles describing the orientation of $\mathbf{m}_{i}$ relative to $x y z$.

network, covering all allowable ranges of the end-to-end vector $\mathbf{r}$. The resulting expression conveniently separates into a single-chain configurational factor and a strain factor as set forth in the introduction and in more detail in ref 1.

Segmental Orientation in a Phantom Network. A phantom network consists of $\nu$ chains joined at $\mu$ junctions of functionality $\phi$. The chains are subject only to constraints imposed by network connectivity. Their dimensions fluctuate in time during which they may pass through each other in a phantomlike manner. Due to this assumption of free interpenetrability, all intermolecular correlations among chains are eliminated. Only the deformations of chains due to the topological connectivity of the network structure are of consequence. The reader is referred to ref 5 for a complete review of the phantom network structure. In the following we assume that the phantom network is under simple tension along the $X$-axis. The state of deformation is defined by the deformation gradient tensor, $\lambda$,

$$
\lambda=\left[\begin{array}{lll}
\left(v_{20} / v_{2}\right)^{1 / 3} \alpha & & \\
& \left(v_{20} / v_{2}\right)^{1 / 3} \alpha^{-1 / 2} & \\
& & \left(v_{20} / v_{2}\right)^{1 / 3} \alpha^{-1 / 2}
\end{array}\right]
$$

where $\alpha$ is the ratio of the final length of the sample to its initial length in the swollen but undistorted state. $v_{2}$ is the volume fraction of polymer in the swollen network during the experiment and $v_{20}$ is the polymer volume fraction during cross-linking. According to the theory of Nagai, ${ }^{3,4}$ the orientation function is given for the phantom network $^{1}$ in terms of the components of $\lambda$ as

$$
S_{x}=D_{0}\left[(1-2 / \phi)\left(v_{20} / v_{2}\right)^{2 / 3}\left(\alpha^{2}-\alpha^{-1}\right)\right]
$$

where

$$
D_{0}=\left(3\left\langle r^{2} \cos ^{2} \Phi\right\rangle_{0} /\left\langle r^{2}\right\rangle_{0}-1\right) / 10
$$

Here, $\Phi$ represents the angle between $\mathbf{m}_{i}$ and $\mathbf{r}$, and the subscript 0 denotes averaging for the free chain. The subscript $x$ appeared to $S$ indicates the direction of stretch. Comparison of eq 4 with eq 1 shows the differences between the Kuhn formulation and the present one. The term in brackets in eq 4 reflects contributions from network structure as well as macroscopic strain. The front factor or the configurational factor, given by eq 5 , equates to $1 / 5 \mathrm{~N}$ only if $\mathbf{m}_{i}$ is taken along the bond direction and the chain is freely jointed. ${ }^{6}$ The reader is referred to ref 1,3 , or 4 for the derivation of eq 4 .

Evaluation of $\boldsymbol{D}_{0}$ for Polymethylene. A short sequence of a polymethylene chain in the all-trans form is shown in Figure 1b. Only the carbon atoms of the backbone are indicated. $i$ denotes the sequential order of a bond situated between the $(i-1)$ st and $i$ th carbon atom. The initial carbon atom at one end of the chain is taken as the zeroth atom. The $x$-axis of the bond-based reference frame is chosen along the bond $i$ and the $y$-axis is in the plane of bonds $i-1$ and $i$, making an acute angle with the extension of bond $i-1$. The $z$-axis completes a righthanded Cartesian coordinate system. Similar bond-based reference frames may be defined for all skeletal bonds. The position of $\mathbf{m}_{i}$ relative to $x y z$ is specified by the two angles, $\phi$ and $\theta . \phi$ is the angle between the $x$-axis and $\mathbf{m}_{i}$. $\theta$ is the angle between the $y$-axis and the projection of $\mathbf{m}_{i}$ on the $y z$ plane.

The configurational average $\left\langle r^{2} \cos ^{2} \Phi\right\rangle_{0}$ appearing in eq 5 is evaluated ${ }^{4,7}$ by the rotational isomeric state scheme according to the expression

$$
\left\langle r^{2} \cos ^{2} \Phi\right\rangle_{0}=\mathcal{F}_{[1}\left(\prod_{k=2}^{i-1} \mathcal{F}^{\prime}{ }_{k}\right) \mathcal{F}_{i}\left(\prod_{k=i+1}^{n-2} \mathcal{F}_{k}{ }_{k}\right) \mathcal{F}_{n]} Z^{-1}
$$

where

$$
\mathscr{F}_{i}=\left(\mathbf{U}_{i} \otimes \mathbf{E}_{26}\right)\left\|\mathbf{F}_{i}\right\|
$$

$\mathbf{U}_{i}$ is the statistical weight matrix, $\mathbf{E}_{26}$ is the identity matrix of order $26, \otimes$ is the direct product, $\left\|\mathbf{F}_{i}\right\|$ is the diagonal array for trans $(t)$, gauche $+\left(\mathrm{g}^{+}\right)$, and gauche $\left(\mathrm{g}^{-}\right)$states for the generator matrix $\mathbf{F}_{i}$ for bond $i$

$$
\left\|\mathbf{F}_{i}\right\|=\left[\begin{array}{lll}
\mathbf{F}_{i}(\mathrm{t}) & & \\
& \mathbf{F}_{i}\left(\mathbf{g}^{+}\right) & \\
& & \mathbf{F}_{i}\left(\mathbf{g}^{\cdot}\right.
\end{array}\right]
$$

with

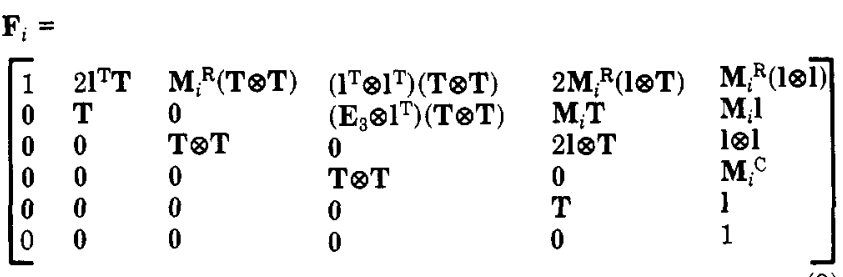

Here, 1 is the bond vector and $\mathbf{T}$ is the transformation tensor from a given bond-based frame to the preceding one. $\mathbf{M}_{i}$ is given by

$$
\mathbf{M}_{i}=\mathbf{m}_{i}^{\mathrm{T}} \otimes \mathbf{m}_{i}
$$

The superscripts $T, R$, and $C$ denote the transpose, row and column forms, respectively. $\mathbf{E}_{3}$ is the identity matrix of order 3 . The primed matrices $\mathfrak{F}_{k}{ }^{\prime}$ in eq 6 are obtained by equating the components of $\mathbf{M}_{i}$ to zero in eq 9 . In eq $6, Z$ denotes the partition function given by

$$
Z=\prod_{i=1}^{n} \mathbf{u}_{i}
$$




$$
\mathbf{U}_{1}=\operatorname{row}(1,0,0) \quad \mathbf{U}_{n}=\operatorname{col}(1,1,1)
$$

and for polymethylene, $\mathbf{U}_{i}$ 's are

$$
\mathbf{U}_{2}=\mathbf{U}_{n-1}=\left[\begin{array}{lll}
1 & 1 & 1 \\
1 & 1 & 0 \\
1 & 0 & 1
\end{array}\right]
$$

and

$$
\mathbf{U}_{i}=\left[\begin{array}{lll}
1 & \sigma & \sigma \\
1 & \sigma & \sigma w \\
1 & \sigma w & \sigma
\end{array}\right] \quad 2<i<n-1
$$

where $\sigma=0.54$ and $w=0.088$ at $140^{\circ} \mathrm{C}$. The terminal matrices $\mathcal{F}_{[1}$ and $\mathcal{F}_{n]}$ in eq 6 are defined by

$$
\mathcal{F}_{[1}=\mathrm{U}_{1} \otimes \mathbf{F}_{[1} \quad \mathcal{F}_{n]}=\mathbf{U}_{n} \otimes \mathbf{F}_{n]}
$$

where [ 1 and $n$ ] appended to $\mathbf{F}$ denote the first row and the last column, respectively. The expression given by eq 6 is for a chain with $\mathbf{m}_{i}$ attached only at bond $i$. If the chain contains several vectors $\mathbf{m}_{i}$ at different bonds, the matrices $\mathcal{F}_{i}^{\prime}$, corresponding to those bonds have to be replaced by $\mathcal{F}_{i}$ containing the vector $\mathbf{m}_{i}$. The mean-squared end-to-end distance $\left\langle r^{2}\right\rangle_{0}$ is evaluated according to matrix generation method as

$$
\left\langle r^{2}\right\rangle_{0}=g_{[1}\left(\prod_{k=2}^{n-2} g_{k}\right) g_{n]} Z^{-1}
$$

where

$$
g_{k}=\left(\mathbf{U}_{k} \otimes \mathbf{E}_{5}\right)\|\mathbf{G}\|
$$

Here, $\mathbf{E}_{5}$ is the identity matrix of order 5 and

$$
\|\mathbf{G}\|=\left[\begin{array}{lll}
\mathbf{G}(\mathrm{t}) & & \\
& \mathbf{G}\left(\mathrm{g}^{+}\right) & \\
& & \mathbf{G}\left(\mathrm{g}^{-}\right)
\end{array}\right]
$$

with

$$
\mathbf{G}=\left[\begin{array}{lll}
1 & 2 \mathbf{1}^{\mathrm{T}} \mathbf{T} & l^{2} \\
\mathbf{0} & \mathbf{T} & 1 \\
0 & \mathbf{0} & 1
\end{array}\right]
$$

where $l$ denotes the magnitude of 1 . For the polymethylene chain $1=\operatorname{col}(1.53,0,0)$, the bond angle is $68^{\circ}$, and the rotational minima for trans, $\mathrm{g}^{+}$, and $\mathrm{g}^{-}$are taken in the present study as $0^{\circ}, 120^{\circ}$, and $-120^{\circ}$, respectively. The formulation presented in the preceding paragraphs follows from the procedure given in ref 7 . It is equivalent to, but more suitable for computer programming than, the previous formulation given in ref 1 . Results of calculations based on the present formulation for the polymethylene chain are presented in the following paragraphs.

Effect of Label Direction Relative to the Bond. The orientational position of the vector $\mathbf{m}_{i}$ relative to a bond $i$ is prescribed by the angles $\phi$ and $\theta$ shown in Figure 1c. Letting the vectors $\mathbf{i}, \mathbf{j}$, and $\mathbf{k}$ represent unit vectors along the $x, y$, and $z$ axes, respectively, the vector $\mathbf{m}_{i}$ may be expressed as

$$
\mathbf{m}_{i}=\cos \phi \mathbf{i}+\sin \phi \cos \theta \mathbf{j}+\sin \phi \sin \theta \mathbf{k}
$$

If $\left\langle r^{2} \cos ^{2} \Phi\right\rangle_{0}$ is written as $\left\langle\left(\mathbf{r} \cdot \mathbf{m}_{i}\right)^{2}\right\rangle_{0}$, where the dot denotes the scalar product, and eq 19 is used, the ratio $\left\langle r^{2} \cos ^{2}\right.$ $\Phi\rangle_{0} /\left\langle r^{2}\right\rangle_{0}$ may be written as

$$
\begin{aligned}
\frac{\left\langle r^{2} \cos ^{2} \Phi\right\rangle_{0}}{\left\langle r^{2}\right\rangle_{0}}= & {\left[\left\langle(\mathbf{r} \cdot \mathbf{i})^{2}\right\rangle \cos ^{2} \phi+\left\langle(\mathbf{r} \cdot \mathbf{j})^{2}\right\rangle \sin ^{2} \phi \cos ^{2} \theta+\right.} \\
\left\langle(\mathbf{r} \cdot \mathbf{k})^{2}\right\rangle \sin ^{2} \phi & \sin ^{2} \theta+2\langle(\mathbf{r} \cdot \mathbf{i})(\mathbf{r} \cdot \mathbf{j})\rangle \sin \phi \cos \phi \cos \theta+ \\
2 & \langle(\mathbf{r} \cdot \mathbf{i})(\mathbf{r} \cdot \mathbf{k})\rangle \sin \phi \cos \phi \sin \theta+ \\
& \left.2\langle(\mathbf{r} \cdot \mathbf{j})(\mathbf{r} \cdot \mathbf{k})\rangle \sin ^{2} \phi \sin \theta \cos \theta\right] /\left\langle r^{2}\right\rangle_{0}(20)
\end{aligned}
$$

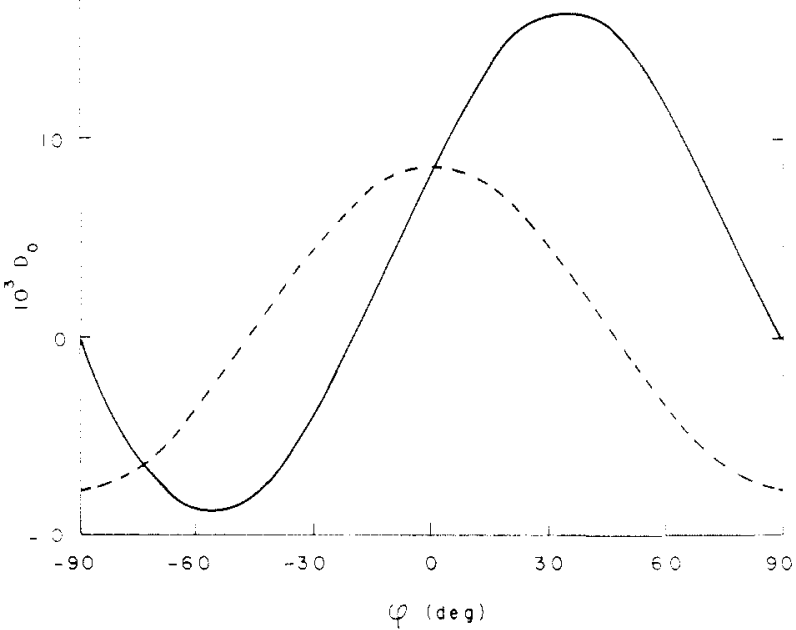

Figure 2. Dependence of $D_{0}$ on $\phi$. Solid line is for $\theta=0$; dashed line is for $\theta=\pi / 2$.

Choosing the vector $\mathbf{m}_{i}$ along the $x$-, $y$-, and $z$-axes of the $i$ th bond frame, the three respective terms $\left\langle(\mathbf{r} \cdot \mathbf{i})^{2}\right\rangle,\left\langle(\mathbf{r} \cdot \mathbf{j})^{2}\right\rangle$, and $\left\langle(\mathbf{r} \cdot \mathbf{k})^{2}\right\rangle$ can be evaluated by the matrix-generation technique described above. The remaining three terms in brackets in eq 20 may be calculated by selecting $\mathbf{m}_{i}$ in the $x y, x z, y z$ planes. Calculations performed for a polyethylene chain of 101 bonds with $\mathbf{m}_{i}$ positioned at the 51st bond lead to

$$
\begin{aligned}
& \frac{\left\langle r^{2} \cos ^{2} \Phi\right\rangle_{0}}{\left\langle r^{2}\right\rangle_{0}}=0.3621 \cos ^{2} \phi+0.3304 \sin ^{2} \phi \cos ^{2} \theta+ \\
& 0.3076 \sin ^{2} \phi \sin ^{2} \theta+0.0785 \sin \phi \cos \phi \cos \theta(20 a)
\end{aligned}
$$

The values of $D_{0}$ obtained by the use of eq $20 \mathrm{a}$ in eq 5 are plotted in Figure 2 for $\theta=0$ (solid curve) and $\theta=90^{\circ}$ (dashed curve) and for various values of $\phi$. Results indicate that orientation measurements are sensitively dependent on the direction of the label relative to the bond axis. The maximum value of 0.0166 for $D_{0}$ is at $\theta=0^{\circ}$ and $\phi=34^{\circ}$, corresponding to a vector that lies along the line joining two alternate carbon atoms. For the simple polymethylene structure, this direction may be identified with the direction of a Kuhn segment as described more fully in the sequel. For asymmetric chains, such an identification fails, however. The value of $D_{0}$ for the direction of a $\mathrm{C}-\mathrm{H}$ bond for which $\theta=60^{\circ}$ and $\phi=124^{\circ}$ is obtained from eq $20 \mathrm{a}$ and 5 as -0.0069 . This result shows that the reorientation of a vector along a $\mathrm{C}-\mathrm{H}$ bond, being negative, differs strongly from that of the chain axis. The same conclusion may be deduced from the work of Flory ${ }^{8}$ for the polybutadiene chain. The negative orientation of the $\mathrm{CH}$ bond of polybutadiene has been experimentally confirmed by Gronski et al. ${ }^{9}$

Effect of Chain Length. Variation of $D_{0}$ with chain length is shown in Figure 3 where the ordinate represents $D_{0}$ and the abscissa is $1 / N$. Calculations are performed with $\mathbf{m}_{i}$ situated at the middle of each chain. The upper solid line shows results for chains in which $\mathbf{m}_{i}$ is directed along a line joining two alternate carbon atoms. The lower solid line is for chains with $\mathbf{m}_{i}$ lying along the $\mathrm{C}-\mathrm{C}$ bond. The curves in Figure 3 may be approximated by straight lines, verifying the inverse relation of $D_{0}$ to chain length. The dashed straight line represents the relation $D_{0}=1 / 5 \mathrm{~N}$ for the Kuhn model of a freely jointed chain.

The ratio $D_{0} /(1 / 5 N)$ is expected to reflect the number of $\mathrm{C}-\mathrm{C}$ bonds in an equivalent Kuhn segment. For the symmetric polymethylene chain, the direction along two 


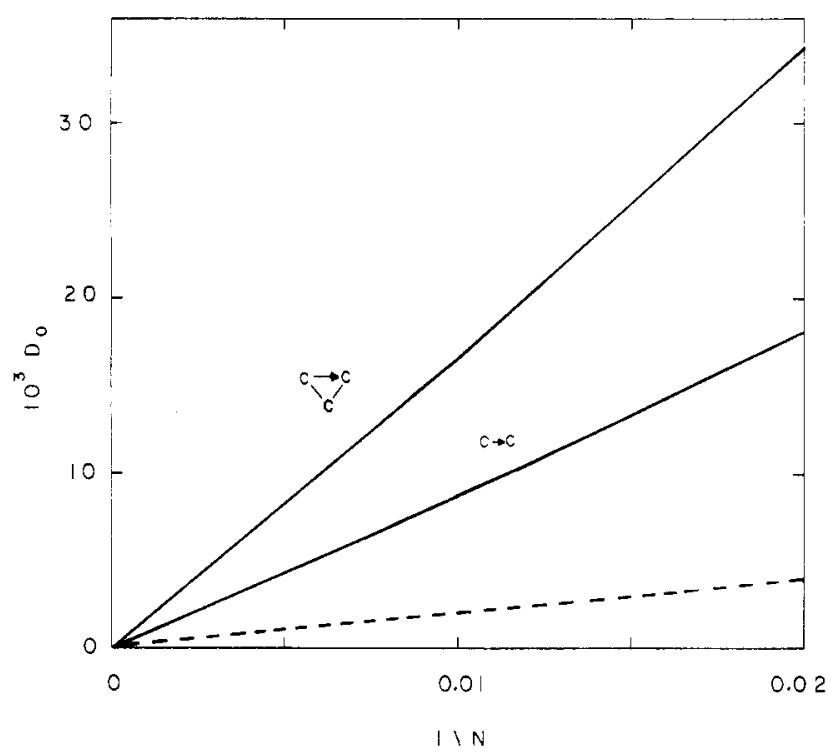

Figure 3. Dependence of $D_{0}$ on $1 / N$. Solid curves are obtained by the matrix-generation technique described in the text. Dashed line is obtained from eq 2.

alternate carbon bonds may be identified with the direction of a Kuhn segment. With this identification, the ratio of the ordinates of the upper solid line to those of the dashed line in Figure 3 may be taken as the required number of bonds in the equivalent Kuhn segment. From the figure, the value of this ratio is 8.3 , which is close to the number $m(=9.7)$ of $\mathrm{C}-\mathrm{C}$ bonds in the Kuhn segment for polymethylene.

The size of the Kuhn length cannot be defined uniquely, however. Detailed statistical analysis for polymethylene ${ }^{10,11}$ and polyoxyalkanes ${ }^{12}$ shows that the equivalent chain selected to reproduce the higher rank even moments differs from the conventional Kuhn equivalent chain. To match the space-configurational characteristics of polymethylene, the parameter $m \approx 20$ is preferred over $m \approx 10^{10-12}$

Effect of Label Location along the Chain. In the calculation of the ratio $\left\langle r^{2} \cos ^{2} \Phi\right\rangle_{0}$, the first two bonds of the chain are kept fixed in the $x y$ plane of the first bond-based coordinate system. A vector $m$ affixed to the first bond of the chain is therefore fixed in space. For long chains, the projection of $\left\langle r^{2}\right\rangle_{0}$ along $\mathbf{m}$ is $\left\langle r^{2}\right\rangle_{0} / 3$, and the ratio $\left\langle r^{2} \cos ^{2} \Phi\right\rangle_{0} /\left\langle r^{2}\right\rangle_{0}$ equates to $1 / 3$ and $D_{0}=0$. Variation of $D_{0}$ with location of label along the chain is shown in Figure 4 for a chain with 101 bonds and $\mathbf{m}_{i}$ being directed along the $\mathrm{C}-\mathrm{C}$ bond $i$. Thus $i$ represents the location of $\mathbf{m}$ along the chain. $D_{0}$ is seen to rapidly reach a constant value as $i$ increases. $D_{0}$ equates to 0.00863 when $n=51$. The average orientation for the chain when all of the $\mathrm{C}-\mathrm{C}$ bonds are assumed to contain the vector $\mathbf{m}_{i}$ is calculated as 0.00805 by using eq $5-18$ with all of the $\mathfrak{F}_{i}^{\prime}$ matrices replaced by $\mathscr{F}_{i}$ in eq 6 .

Effect of Temperature. Changes in temperature affect the statistics of the chain by modifying the parameters $\sigma$ $=\exp \left(-E_{\sigma} / R T\right)$ and $w=\exp \left(-E_{\mathrm{w}} / R T\right)$ where $E_{\sigma}$ is the energy of the gauche state relative to trans and $E_{\mathrm{w}}$ is the energy of the $\mathrm{g}^{ \pm} \mathrm{g}^{\mp}$ state in excess of $2 E_{\sigma}$ (see eq 13). $D_{0}$ is expected to be affected by such changes depending on the direction of the vector $\mathbf{m}$ relative to bond. Results of calculations for the polymethylene chain of 101 bonds with $\mathbf{m}$ affixed at the middle bond are shown in Figure 5 for a temperature range of $100 \mathrm{deg}$. The effect of temperature on $D_{0}$ in this range is within $10 \%$. If larger variations in $D_{0}$ are observed experimentally they should be attributed to effects of intermolecular correlations on the orientation of the vector $\mathbf{m}$.

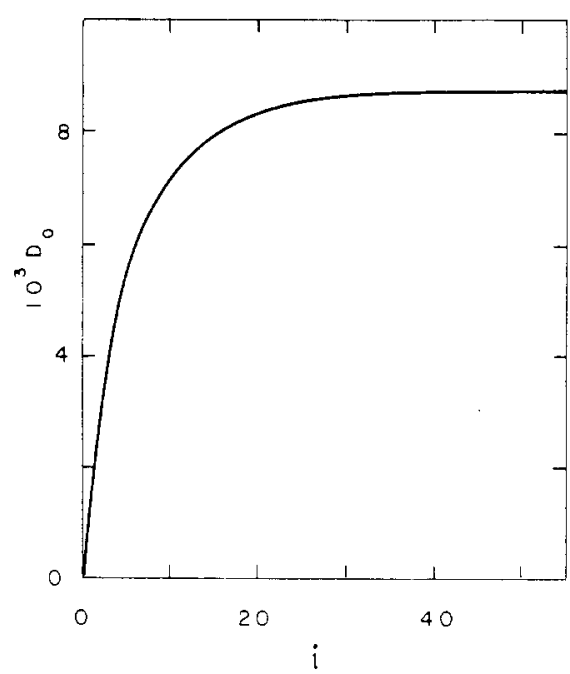

Figure 4. Dependence of $D_{0}$ on the location of label on the chain for a polymethylene chain with 101 bonds. $i$ along the abscissa denotes the index of bond with $\mathbf{m}_{i}$.

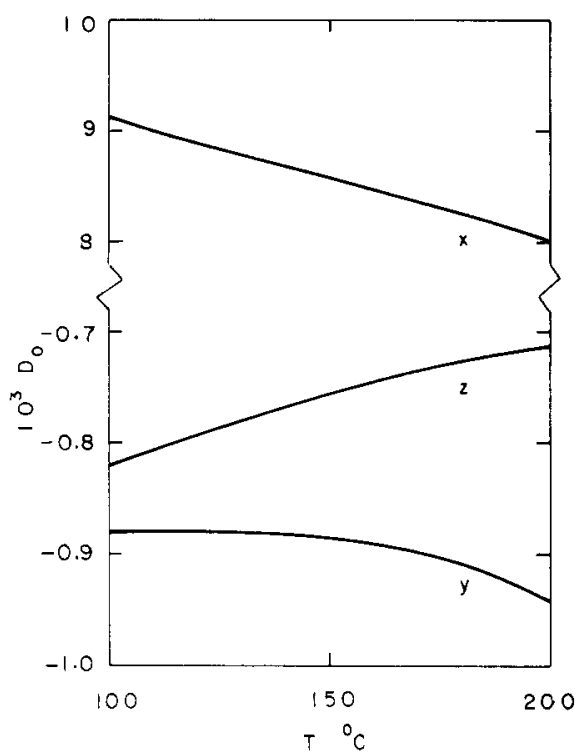

Figure 5. Dependence of $D_{0}$ on temperature. $x, y$, and $z$ denote the direction of $\mathbf{m}_{i}$.

Effect of Configurational Perturbations Due to the Presence of a Label. Calculations for the polymethylene chain show that the value of $D_{0}$ depends sensitively on the local configurational properties of bonds around the label vector $\mathbf{m}_{i}$. Such local configurational perturbations may possibly occur by the incorporation of bulky groups such as dimethylanthracene into the chain required for fluorescence experiments. In order to investigate the effects of such perturbations, two bonds on each side of the 51 st bond of a 101 bond chain are fixed in the trans configuration. Exclusion of the $\mathrm{g}^{+}$and $\mathrm{g}^{-}$configurations in this manner is expected to induce the "stiffening" effect of a bulky label. Calculations show that $D_{0}$ for a vector along the $\mathrm{C}-\mathrm{C}$ bond changes from 0.00863 to 0.01376 , indicating an increase of about $60 \%$ over the unperturbed value. This sensitive dependence of $D_{0}$ on local perturbations emphasizes the necessity for correcting for the perturbation of the orientational behavior of the chain, caused by the presence of the label when interpreting experimental results.

Effect of Polydispersity. Results of calculations presented in Figure 3 show that $D_{0}$ varies inversely with chain length. This is also indicated in the Kuhn formula given by eq 1 . 
Thus, for a monodisperse system of chains with molecular weight $M$,

$$
D_{0}=k / N=k M_{\mathrm{u}} / M
$$

where $k$ is the constant of proportionality and $M_{\mathrm{u}}$ is the molecular weight of the repeat unit. When chains of different sizes are present, eq 21 has to be replaced by

$$
\bar{D}_{0}=k M_{\mathrm{u}} / \bar{M}
$$

where $\bar{D}_{0}$ is the averaged front factor and $\bar{M}$ is a properly averaged molecular weight. Eliminating the term $k M_{\mathrm{u}}$ among eq 21 and 21 a leads to

$$
\bar{D}_{0}=(M / \bar{M}) D_{0}
$$

Inasmuch as the value of $D_{0}$ for a unimodal system can be evaluated by the procedures described above, eq 22 serves as a convenient way of calculating the averaged factor $\bar{D}_{0}$ for a system comprising different chain lengths. The average $\bar{M}$, however, depends on the type of labeling.

The dependence of $\bar{D}_{0}$ on $\bar{M}$ given by eq 22 is based on the assumption that short and long chains experience the same amount of deformation in the network. Recent experimental evidence ${ }^{20}$ from bimodal PDMS networks suggests that longer chains may be deforming more than the shorter ones. The dependence of molecular deformation on chain length should then be reflected in eq 22 . The solution to the problem awaits further experimental evidence on the mode of chain deformation in polydisperse networks.

\section{Contribution from Network Topology and Intermolecular Correlations to Segmental Orientation in a Real Network}

The hypothetical phantom network model fully reflects the contributions from chain configurations to the orientation function through the front factor $D_{0}$. In real networks, intermolecular correlations and contributions from constraints are two major factors that complicate the picture. The expression for the orientation function in a real network in simple tension is given as

$$
\begin{aligned}
S= & \left(D_{0}+D_{\text {int }}\right)(1-2 / \phi)\left(v_{20} / v_{2}\right)^{2 / 3}\left[\alpha^{2}-\alpha^{-1}+\right. \\
& \left.2\left(v_{2} / v_{20}\right)^{2 / 3}(\phi-2)^{-1}\left(B_{1}-B_{2}+(\phi e / 2)\left(g_{1} B_{1}-g_{2} B_{2}\right)\right)\right]
\end{aligned}
$$

Here, $D_{\text {int }}$ represents the intermolecular contribution to orientation. It results from orientational correlations among sequences of neighboring molecules. $B_{t}$ and $g_{t}$ are defined as

$$
\begin{gathered}
B_{t}=\left(\lambda_{t}-1\right)\left(\lambda_{t}+1-\zeta \lambda_{t}{ }^{2}\right) /\left(1+g_{t}\right)^{2} \\
g_{t}=\lambda_{t}{ }^{2}\left[\kappa^{-1}+\zeta\left(\lambda_{t}-1\right)\right]
\end{gathered}
$$

with $t=1$ or $2 . k$ and $\zeta$ in eq 24 and 25 are the parameters of the constrained junction model of rubber elasticity. ${ }^{13}$ The entanglement of chains in a network arising from effects of chain incrossability is represented by elastic constraints operating on the fluctuations of junctions. This elastic action of constraints depends on strain and hence contributes further to the orientation of segments. The coupling of the segments to the domains of constraints defined in this manner is represented by the "coupling parameter" $e$ in eq 23.

The experimental identification of the various parameters introduced in eq 23 over those given by eq 4 is of major importance for quantitative characterization of orientation in real networks.

Experiments have shown that segmental orientation decreases considerably upon swelling of the network. ${ }^{2}$ Calculations, on the other hand, show that the term in brackets in eq 23 remains approximately constant with swelling. This observation indicates that $D_{\text {int }}$ depends sensitively on the degree of swelling, as expected. Experiments have also shown ${ }^{2}$ a strong decrease in orientation with increasing temperature. This dependence on temperature may be attributed mainly to $D_{\text {int }}$ inasmuch as calculations in the preceding section indicate that $D_{0}$ is relatively insensitive to temperature. Swelling and heating may thus be employed to eliminate $D_{\text {int }}$ to an appreciable extent. Contributions from constraints persist in eq 23, however. This necessitates a careful characterization of the network parameters, $\kappa, \zeta$, and $e$.

\section{Discussion}

Results of calculations of segmental orientation in deformed networks presented in this study point to various aspects of the phenomenon that is relevant to experimental work in this field. Elimination of intermolecular correlations by swelling with an isotropic solvent and by making measurements at elevated temperatures seems to be of major importance. That the contribution to orientation by $D_{\text {int }}$ may be several times that of $D_{0}$ has been shown by previous experiments on different systems. ${ }^{2,14}$ On more specific grounds, swelling should be employed in the wide-angle X-ray scattering analysis of networks to eliminate contributions from intermolecular sources. In the absence of intermolecular contributions, the wide-angle $\mathrm{X}$-ray scattering technique is especially convenient for measuring segmental orientation in deformed networks. ${ }^{15}$

Recent deuterium NMR experiments of Deloche and collaborators ${ }^{16-18}$ show that this technique is suitable for measurements of segmental orientation in networks. The accuracy and reproducibility of measurements over wide ranges of deformation, including very small deformations, are remarkable. The accuracy of the technique may lead to a precise test of the molecular considerations brought up in the present paper.

The effect of label orientation relative to repeat unit seems to be equally important according to the present analysis. Corrections to account for the orientation of a label not directed along the backbone bond are performed by Gronski et al., ${ }^{9}$ assuming that the end-to-end vector of a certain statistical segment is uncorrelated (orientationally) with the label vector. Predictions based on this correction seem to account for the negative orientation of the C-D bond in stretched polybutadiene. ${ }^{9}$ The method, however, is approximate due to the assumption of uncorrelated configurations at the local level. The present treatment is rigorous within the limits of the rotational isomeric state theory and is aimed at removing the approximations of this nature.

The variation of $D_{0}$ with location on a chain as shown in Figure 4 may be used to separate intermolecular contributions to orientation from intramolecular effects. According to analysis, the $D_{0}$ value for labels situated at the cross-links is zero. Thus, contributions from intermolecular effects only should cause the orientation of these labels. According to experimental evidence cited above, concerning $D_{\text {int }}$, the orientation of cross-link labels should vanish upon swelling with a suitable diluent.

Contributions to segmental orientation in a real network from the excess deformation of chains due to their diffuse entanglement with their neighborhoods is of considerable magnitude. Previous calculations ${ }^{1}$ and comparison with experiments ${ }^{2}$ show that segmental orientation in a real network may be as large as twice that of the phantom network. As stated above, a molecular network model is required for the analysis of contributions from network constitution. The constrained junction model adopted in eq 23 to describe orientation in a real network introduces 
three parameters, $\kappa, \zeta$, and $e$. Stress-strain experiments ${ }^{19}$ show that $\kappa$ may be estimated from the molecular constitution of the network and that $\zeta$ may be taken as zero in the first approximation. Thus, the coupling parameter $e$ appearing in eq 23 is the only parameter that requires evaluation by experiments.

Acknowledgment. We are indebted to Professor L. Monnerie for helpful discussions.

Registry No. Polymethylene, 25038-57-7.

\section{References and Notes}

(1) Erman, B.; Monnerie, L. Macromolecules 1985, 18, 1985.

(2) Queslel, J. P.; Erman, B.; Monnerie, L. Macromolecules 1985, $18,1991$.

(3) Nagai, K. J. Chem. Phys. 1964, 40, 2818

(4) Flory, P. J. Statistical Mechanics of Chain Molecules; Interscience Publishers: New York, 1969.
(5) Flory, P. J. Proc. R. Soc. London, A 1976, 351, 351.

(6) Erman, B.; Monnerie, L. Polym. Commun. 1985, 26, 167.

(7) Flory, P. J. Macromolecules 1974, 7, 381.

(8) Flory, P. J.; Abe, Y. Macromolecules 1969, 2, 335.

(9) Gronski, W.; Stadler, R.; Jacobi, M. M. Macromolecules 1984, 17,741 .

(10) Yoon, D. Y.; Flory, P. J. J. Chem. Phys. 1974, 61, 5366.

(11) Jernigan, R. L.; Flory, P. J. J. Chem. Phys. 1969, 50, 4185.

(12) Abe, A.; Kennedy, J. W.; Flory, P. J. J. Polym. Sci., Polym. Phys. Ed. 1976, 14, 1337.

(13) Flory, P. J.; Erman, B. Macromolecules 1982, 15, 800

(14) Erman, B.; Flory, P. J. Macromolecules 1983, 16, 1607.

(15) See, for example: Mitchell, G. R. J. Polym. Sci., Polym. Lett. 1983, 21, 341; Polymer 1985, 26, 1605.

(16) Dubault, A.; Deloche, B.; Herz, J. Polymer 1984, 25, 1405.

(17) Toriumi, H.; Deloche, B.; Herz, J.; Samulski, E. T. Macromolecules $1985,18,304$.

(18) Deloche, B.; Dubault, A.; Herz, J.; Lapp, A. Europhys. Lett. $1986,1,629$.

(19) Erman, B.; Flory, P. J. Macromolecules 1982, 15, 806.

(20) Stein, R. S., private communication.

\title{
Network Structure in Epoxies. 5. Deformation Mechanism in Epoxies
}

\author{
Wen-li Wu* and Barry J. Bauer \\ Polymers Division, Institute for Materials Science and Engineering, National Bureau of \\ Standards, Gaithersburg, Maryland 20899. Received February 25, 1987
}

\begin{abstract}
Small-angle neutron scattering (SANS) was used to investigate the response of network molecules to large strain deformation. Partially deuteriated diglycidyl ether of bisphenol A (DGEBA) was cured with either tri- or diamines of different molecular weights. The change of the average distance between cross-links along the epoxy and the amine linkages can easily be detected from the shift in the positions of the scattering maxima. It was found that the average distance between cross-links remained almost unchanged along both the amine and the epoxy linkages as the bulk material underwent a large deformation. The above results are discussed and compared to the results from swollen networks that do show a substantial increase in average distance between cross-links.
\end{abstract}

\section{Introduction}

The conformational changes in molecules in cross-linked polymers have been a subject of extensive studies for decades. The cross-link density of the materials included in their work was low in most cases. ${ }^{1-4}$ Small-angle neutron scattering (SANS) has proven to be a powerful technique for this type of study. The displacement of the cross-links in response to macroscopic deformation was found to be, in general, between what is predicted by the junction affine and the phantom network models. ${ }^{5-10}$ Materials of high cross-link density such as most thermosetting polymers have not been included in this type of study. This work represents the first effort in applying SANS to study the deformation behavior of a commonly used thermosetting polymer/epoxy.

There are two additional characteristics of the networks in this work that differ from studies in the past. First, there is a difference in the chemical composition between the linkages, and second, there is low functionality of the cross-links. Usually, the linkages in epoxies are composed of two types of chains, one is the epoxy chain and the other is the amine chain. In the present case, the epoxy chain is basically the bisphenol A linkage and the amine one is made of the polypropylene oxide (PPO) linkage. The low functionality of the cross-links also make epoxies an interesting subject of study. Linear epoxies cured with either di- or triamines have a functionality of three. The amine ends that have reacted with two epoxide groups are the only cross-linking points for linear diamines. For triamine cured materials, in addition to the amine end cross-linking points, the junctions at the center of each amine constitutes anther cross-linking points. However, the functionality of this junction is also three.

For linear polymers, deformation results in a change of the overall shape of an average molecule. For example, the radius of gyration, $R_{\mathrm{g}}$, will be distorted from that of a random coil. However the length of each repeat unit remains unchanged, and the change in the value of $R_{\mathrm{g}}$ is caused by the change in average step direction. For a highly cross-linked material; the molecular weight between cross-links is small enough to be considered as on statistical segment of the size commonly used to describe linear chain polymers. For the case of a cross-link functionality of three, it is possible that an average molecular network can accomodate the macroscopic deformation by some mechanism requiring less energy than altering the average distance between cross-links. With a functionality of three, the topological connectivity on a local scale can be considered as two dimensional. Consequently, a network could easily deform by unfolding without a dramatic change in the distance between cross-links. ${ }^{11,12}$ The idea of network unfolding was originally proposed for cross-linked polymers with relatively low cross-linking density, i.e. high population of physical or spatial neighbors. This is not the case 